\title{
Plasma level of D-dimer accompanying different types of gynecologic surgery and effects of prophylactic subcutaneous injection of heparin calcium
}

\author{
Sakika Yanai ${ }^{1}$, Yusuke Nakano ${ }^{2}$, Ken-ichi Honda ${ }^{3}$, Tomoko Sumikura $^{4}$, \\ Saori Seo ${ }^{1}$, Yutaka Inoue ${ }^{3}$, Naohiko Umesaki ${ }^{2}$
}

\author{
${ }^{1}$ Department of Obstetrics \& Gynecology, Osaka City University Graduate School of Medicine, 1-4-3, Asahimachi- \\ 545-8585, Osaka, Japan \\ ${ }^{2}$ Department of Gynecology, Izumi Municipal Hospital, 4-10-10, Huchucho, Izumi-594-0071, Osaka, Japan \\ ${ }^{3}$ Department of Obstetrics \& Gynecology, Kashiwara Municipal Hospital, 1-7-9, Houzenji, Kashiwara-582-0005, \\ Osaka, Japan \\ ${ }^{4}$ Division of Gynecology, National Cancer Center Hospital, Kashiwanoha, Kashiwa-277-8577, Chiba, Japan
}

Received: 27 March 2015

Accepted: 19 April 2015

\section{*Correspondence:}

Dr. Ken-ichi Honda,

E-mail: kenhonda@med.osaka-cu.ac.jp

Copyright: (c) the author(s), publisher and licensee Medip Academy. This is an open-access article distributed under the terms of the Creative Commons Attribution Non-Commercial License, which permits unrestricted non-commercial use, distribution, and reproduction in any medium, provided the original work is properly cited.

\begin{abstract}
Background: The standard range of D-dimer level associated with each type of gynecologic surgery is required to note the occurrence of bleeding or thromboembolism.

Methods: Plasma levels of D-dimer of patients who underwent different types of gynecologic surgery were measured on the Day of Preoperative Examination (DPE) and the first postoperative day (POD-1). Patients were classified by surgery type: hysterectomy for benign diseases or cervical intraepithelial neoplasia; hysterectomy for uterine cancer; surgery for ovarian cancer; laparoscopic surgery for a benign adnexal mass; laparotomy for a benign adnexal mass; laparotomic myomectomy; cervical conization; transcervical resection of an intrauterine mass; vaginal surgery for prolapse of a pelvic organ.

Results: In each type of gynecologic surgery, plasma levels of D-dimer on POD-1 were higher than those on the DPE. Prophylactic subcutaneous injection of heparin calcium for patients who underwent surgery for endometrial cancer showed no significant difference in the plasma level of D-dimer on the sixth postoperative day (POD-6) and the plasma level of D-dimer on POD-6 was in the same level as those on POD-1.

Conclusions: Plasma levels of D-dimer on POD-1 were higher than those on the DPE in each type of gynecologic surgery. The D-dimer level remained high even on POD-6, and not changed by prophylactic subcutaneous injection of heparin calcium.
\end{abstract}

Keywords: D-dimer, DPE, POD-1, Gynecologic surgery, Heparin calcium

\section{INTRODUCTION}

D-dimer is a specific antigen of fibrin degradation products, and has been used for the exclusion of venous thromboembolism (VTE). ${ }^{1}$ In clinical practice for postoperative patients, the plasma level of D-dimer is elevated by the effects of surgery. ${ }^{2}$ We tried to ascertain the standard plasma range of D-dimer on postoperative patients for evaluation of abnormal bleeding or VTE after gynecologic surgery. 


\section{METHODS}

The plasma level of D-dimer in patients who underwent gynecologic surgery at the Izumi Municipal Hospital (Izumi, Japan) from July 2011 to March 2014 was measured on the Day of Preoperative Examination (DPE) and the first postoperative day (POD-1). Citrated plasma was collected from patient blood and analyzed for Ddimer concentration using anti-human D-dimer mouse monoclonal antibody-coated latex (Nanopia D-dimer; Sekisui Medical, Tokyo, Japan).

Patients were classified by surgery type; hysterectomy for benign diseases or Cervical Intraepithelial Neoplasia (CIN); hysterectomy for uterine cancer; surgery for ovarian cancer; laparoscopic surgery for a benign adnexal mass; laparotomy for a benign adnexal mass; laparotomic myomectomy; cervical conization; transcervical resection of an intrauterine mass; vaginal surgery for prolapse of a pelvic organ.

Plasma levels of D-dimer of patients who underwent surgery for endometrial cancer with or without postoperative subcutaneous injection of heparin calcium (5000 units twice daily for 5 days, Mochida Pharmaceutical Co. Tokyo, Japan) were measured on the DPE, POD-1, and the sixth postoperative day (POD-6). Data were analyzed by the Student's t-test using SPSS v18 (IBM, Armonk, NY, USA).

\section{RESULTS}

In 113 patients who underwent gynecologic surgery including hysterectomy for benign disease or CIN, the Ddimer level on POD-1 (3.44 $\pm 3.06 \mathrm{ng} / \mathrm{mL}$ (mean \pm standard deviation)) was significantly higher $(\mathrm{P}<0.001)$ than the preoperative level of D-dimer $(0.73 \pm 0.61 \mathrm{ng} / \mathrm{mL})$ (Figure 1). In 70 patients who underwent surgery including hysterectomy for uterine cancer, the D-dimer level on POD-1 $(5.81 \pm 4.55 \mathrm{ng} / \mathrm{mL})$ was significantly higher $(\mathrm{P}<0.001)$ than the preoperative $\mathrm{D}$-dimer level ' $(1.11 \pm 1.48 \mathrm{ng} / \mathrm{mL})$. In 36 patients who underwent surgery including hysterectomy for ovarian cancer, the Ddimer level on POD-1 (7.89 $\pm 6.56 \mathrm{ng} / \mathrm{mL})$ was significantly higher $(\mathrm{P}<0.001)$ than the preoperative $\mathrm{D}$ dimer level $(4.01 \pm 5.61 \mathrm{ng} / \mathrm{mL})$.

In 22 patients who underwent laparotomy for an adnexal benign mass, the D-dimer level on POD-1 (2.52 \pm 1.96 $\mathrm{ng} / \mathrm{mL})$ was significantly higher $(p<0.001)$ than the preoperative D-dimer level $(0.69 \pm 0.44 \mathrm{ng} / \mathrm{mL})$ (Figure 2). In 38 patients who underwent laparoscopic surgery for an adnexal benign mass, the D-dimer level on POD-1 $(2.14 \pm 1.85 \mathrm{ng} / \mathrm{mL})$ was significantly higher $(\mathrm{P}<0.001)$ than the preoperative D-dimer level $(0.57 \pm 0.48 \mathrm{ng} / \mathrm{mL})$. In eight patients who underwent laparotomic myomectomy, the D-dimer level on POD-1 $(9.89 \pm 5.21$ $\mathrm{ng} / \mathrm{mL})$ was significantly higher $(\mathrm{P}=0.001)$ than the preoperative D-dimer level $(0.36 \pm 0.30 \mathrm{ng} / \mathrm{mL})$.

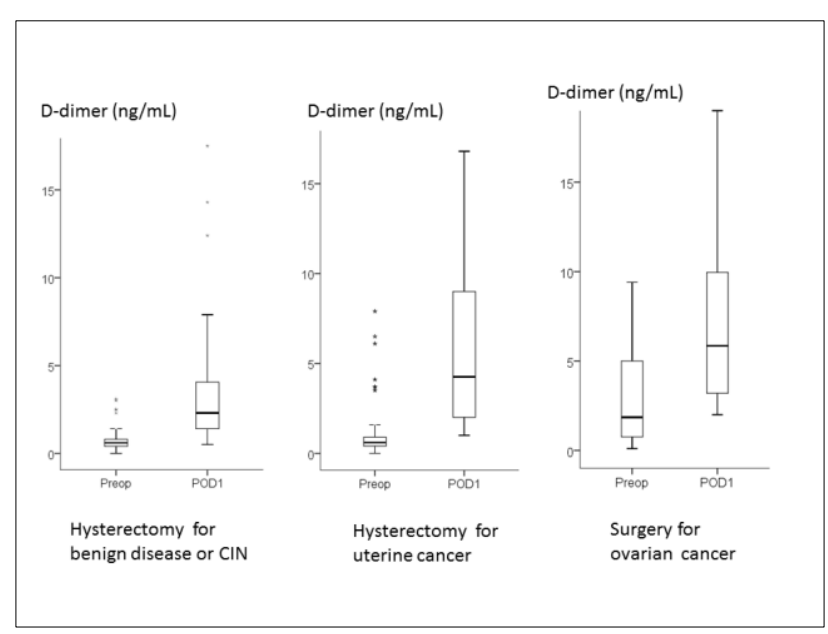

Figure 1: D-dimer level in plasma on the DPE and POD-1 for hysterectomy for benign disease or CIN, hysterectomy for uterine cancer, and surgery for ovarian cancer.

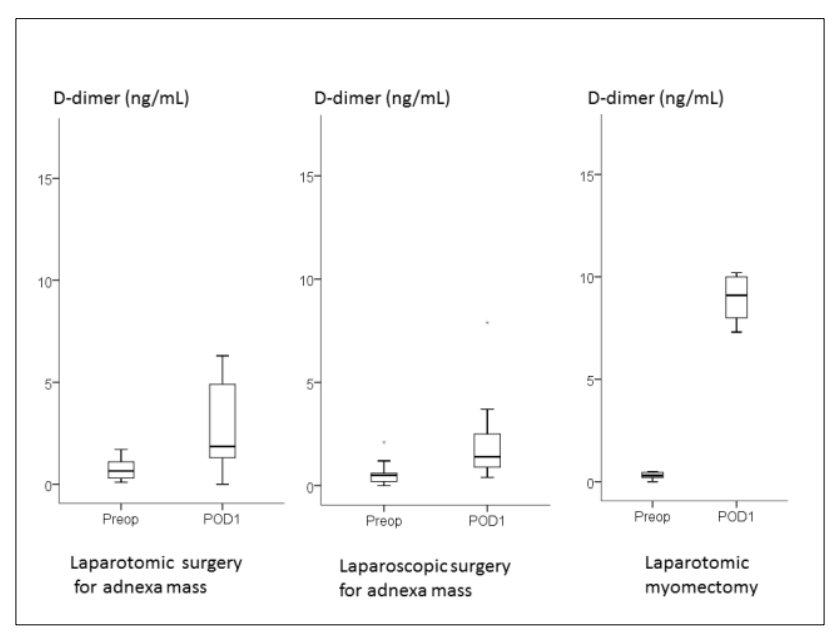

Figure 2: D-dimer level in plasma on the DPE and POD-1 for laparotomic or laparoscopic surgery for an adnexal mass or laparotomic myomectomy.

In 36 patients who underwent vaginal surgery, the Ddimer level on POD-1 (3.01 $\pm 4.03 \mathrm{ng} / \mathrm{mL})$ was significantly higher $(\mathrm{p}=0.002)$ than the preoperative $\mathrm{D}$ dimer level $(0.93 \pm 0.68 \mathrm{ng} / \mathrm{mL}$ ) (Figure 3$)$. In 17 patients who underwent transcervical resection of a uterine mass, the D-dimer level on POD-1 $(0.88 \pm 0.47 \mathrm{ng} / \mathrm{mL})$ was significantly higher $(\mathrm{P}=0.007)$ than the preoperative $\mathrm{D}$ dimer level $(0.59 \pm 0.42 \mathrm{ng} / \mathrm{mL})$. In 31 patients who underwent conization for CIN, the D-dimer level on POD-1 $(0.67 \pm 0.37 \mathrm{ng} / \mathrm{mL})$ was significantly higher $(\mathrm{P}=$ $0.01)$ than the preoperative $\mathrm{D}$-dimer level $(0.46 \pm 0.27$ $\mathrm{ng} / \mathrm{mL})$.

Of 13 patients who underwent laparotomic hysterectomy, bilateral adnexectomy, or lymphadenectomy for endometrial cancer, seven patients (heparin group) were evaluated after subcutaneous injection of heparin calcium, and six patients (control group) were evaluated 
without injection of an anticoagulant (Figure 4). The Ddimer level of each group was not significantly different between heparin group and control group on the DPE (P $=0.608 ; 0.70 \pm 0.28$ vs. $0.92 \pm 0.59)$, $\mathrm{POD}-1(\mathrm{P}=0.429$; $5.08 \pm 3.56$ vs. $4.23 \pm 2.68)$, and POD-6 $(\mathrm{P}=0.275 ; 6.22$ \pm 3.45 vs. $3.83 \pm 2.46$ ), although the mean D-dimer level was lower in the heparin group compared with the control group. The D-dimer level on POD-6 remained higher than on the DPE and there was no significant difference between on POD-1 and POD-6 in either group. There was no occurrence of VTE in these 13 patients.

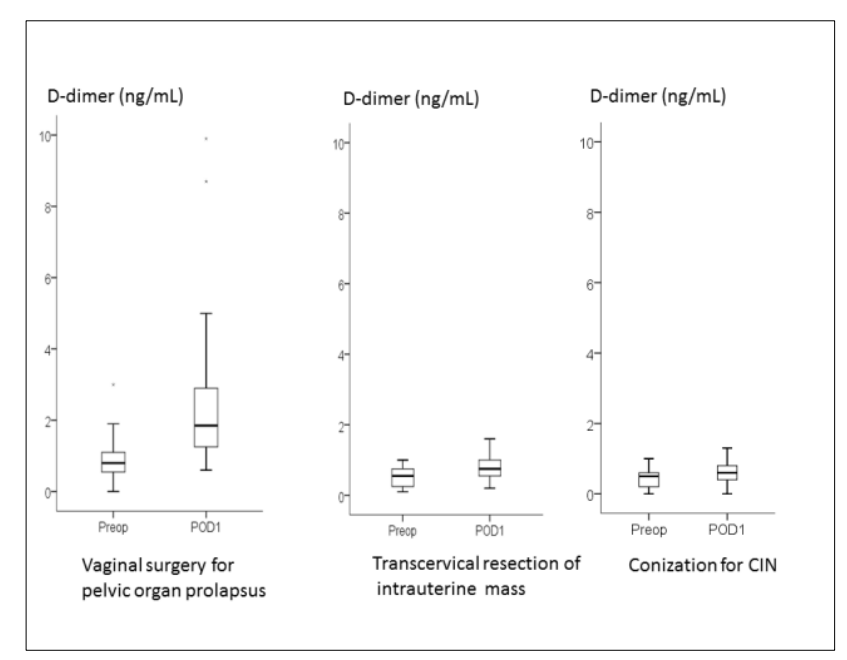

Figure 3: D-dimer level in plasma on the DPE and POD-1 for vaginal surgery for prolapse of a pelvic organ, transcervical resection of intrauterine mass, or conization for CIN.

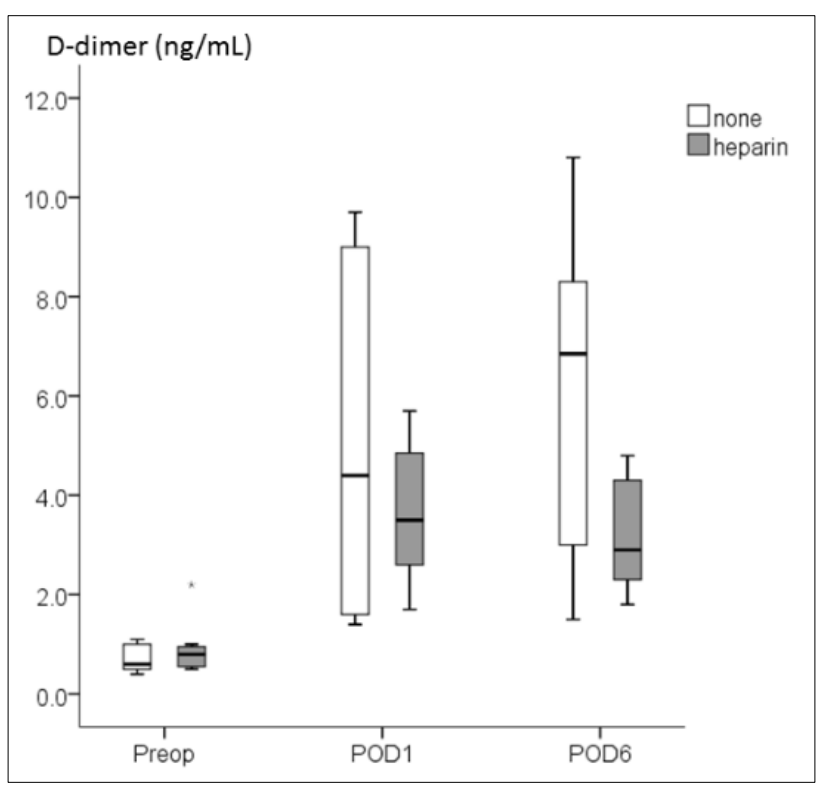

Figure 4: Effects of postoperative subdermal injection of heparin calcium on plasma levels of D-dimer in patients who underwent hysterectomy, bilateral adnexectomy, or lymphadenectomy for endometrial cancer.

\section{DISCUSSION}

D-dimer is an antigen of the degradation product of crosslinked fibrin. It is used as a marker to exclude deep venous thrombosis, but a positive result does not suggest thrombosis because D-dimer has low specificity. However, D-dimer values on POD-1 seem to differ depending on how wide an area the surgical procedures induce fibrin formation. ${ }^{3}$ A D-dimer level that is higher than the normal level for surgery may suggest fibrin formation over a wide area or venous thrombosis.

In all gynecologic surgery, from conization of the cervix to extended surgery for ovarian cancer, the D-dimer level on POD-1 was significantly higher than that on the DPE, though the D-dimer level showed a different distribution range depending on surgery type. The range of the plasma level of D-dimer on POD-1 was low for cervical conization and transcervical resection, but high for laparoscopic resection of an adnexal benign mass, vaginal surgery for prolapse of a pelvic organ, and laparotomic surgery with or without hysterectomy. After myomectomy, the plasma level of D-dimer was markedly higher on POD-1 than for other types of gynecologic surgery.

In patients with uterine cancer or those with ovarian cancer, the mean level of D-dimer in plasma on the DPE was higher than the normal cutoff level $(0-1.0 \mathrm{ng} / \mathrm{mL})$. Mean levels of D-dimer in plasma on POD-1 of patients with ovarian cancer or with uterine cancer were higher than those for patients with benign diseases. We hypothesize that such high elevations of levels of Ddimer reflect hyper-coagulation from malignant tumors and the surgical site.

As anticoagulant prophylactic therapy for postoperative pulmonary thromboembolism, heparin calcium was injected subcutaneously after blood sampling on POD-1. There was no significant difference in D-dimer level on POD-6 between the control group and heparin group, and no patient experienced thromboembolism in either group. Differences in D-dimer levels between these groups may have been too small to be detected postoperatively as hyper-coagulation or as a large hemostatic area in the surgical wound.

Plasma D-dimer level is reported to be elevated at 150 min from start of surgery, but not yet at 75 min during reduction mammoplasty. ${ }^{4}$ In total knee replacement surgery, it is reported the plasma D-dimer value is elevated during usage of tourniquet, elevated more on POD-1 and returned to normal range on POD-3. In nonsurgical management of gastrointestinal ulcer bleeding, D-dimer level reflects fibrinolytic area on ulcer tissue and medication decreases D-dimer level. ${ }^{6}$

We wished to ascertain the standard range of D-dimer level associated with each type of gynecologic surgery. We are expecting a D-dimer level beyond the standard 
range may suggest postoperative bleeding in the surgical wound or occurrence of VTE.

\section{CONCLUSION}

Plasma levels of D-dimer on POD-1 were higher than those on the DPE in any type of gynecologic surgery. Prophylactic subcutaneous injection of heparin calcium for patients who underwent surgery for endometrial cancer showed no significant difference in plasma levels of D-dimer on POD-6 which remain in the same level as those on POD-1.

\section{ACKNOWLEDGMENTS}

We are grateful for the technical assistance by Mari Yamada, Tsuyoshi Maezono and other staff of the Clinical Laboratory of Izumi Municipal Hospital.

Funding: No funding sources

Conflict of interest: None declared

Ethical approval: Not required

\section{REFERENCES}

1. Adam SS, KeyNigel NS, Greenberg CS. D-dimer antigen: current concepts and future prospects. Blood. 2009;113:2878-87.

2. Davis JD. Prevention, diagnosis, and treatment of venous thromboembolic complications of gynecologic surgery. Am J Obstet Gynecol. 2001;184:759-75.

3. Kodama J, Seki N, Fukushima C, Kusumoto T, Nakamura K, Hiramatsu Y. Postoperative decreased levels of D-dimer in patients with genecologic cancer with enoxaparin and fondaparinux thromboprophylaxis. Molec Clin Oncol. 2013 Jul;1(4):737-44.

4. Payen JF, Baruch M, Horvilleur E, Richard M, Gariod T, Polack B. Changes in specific markers of haemostasis during reduction mammoplasty. $\mathrm{Br} \mathbf{J}$ Anaesth. 1998;80:464-6.

5. Hughes SF, Hendricks BD, Edwards DR, Bastawrous SS, Middleton JF. Lower limb orthopaedic surgery results in changes to coagulation and non-specific inflammatory biomarkers, including selective clinical outcome measures. Eur J Med Res. 2013;18:40.

6. Vreeburg EM, Levi M, Rauws EAJ, Deventer SJHV, Snel P, Bartelsman JWFM, et al. Enhanced mucosal fibrinolytic activity in gastroduodenal ulcer haemorrhage and the beneficial effect of acid suppression. Aliment Pharmacol Ther. 2001;15:63964.

DOI: $10.18203 / 2320-1770 . i j r c o g 20150049$

Cite this article as: Yanai S, Nakano Y, Honda K, Sumikura T, Seo S, Inoue Y, Umesaki N. Plasma level of D-dimer accompanying different types of gynecologic surgery and effects of prophylactic subcutaneous injection of heparin calcium. Int $\mathbf{J}$ Reprod Contracept Obstet Gynecol 2015;4:551-4. 